\title{
Adrenal Tuberculosis Mimicking a Malignant Tumor with Primary Adrenal Insufficiency
}

\author{
Woo Seok Kim, Jin Hyung Lee \\ Department of Surgery, Soonchunhyang University Gumi Hospital, Soonchunhyang University College of Medicine, Gumi, Korea
}

\begin{abstract}
Adrenal tuberculosis is a relatively infrequent cause of primary adrenocortical insufficiency in developed countries. Isolated adrenal tuberculosis, especially with enlargement of adrenal glands, can cause diagnostic problems and requires differentiation from primary or secondary neoplasms. A 40-year-old woman presented to Soonchunhyang University Gumi Hospital with altered consciousness. She showed biochemical evidence of adrenal insufficiency without signs of tuberculosis. Abdominal computed tomography (CT) scan showed a calcified mass $3.3 \times 2.3 \mathrm{~cm}$ in size and peripheral enhancement of the left adrenal gland. A laparoscopic adrenalectomy was performed for a diagnosis of left adrenal malignant tumor. The diagnosis of adrenal tuberculosis was confirmed by a final histological examination.
\end{abstract}

Keywords: Adrenal glands; Tuberculosis; Adrenal insufficiency; Laparoscopy

\section{INTRODUCTION}

Adrenal insufficiency was first described by Thomas Addison in 1855. At that time, the major cause of adrenal gland insufficiency was Mycobacterium tuberculosis infection. Today, in developed countries, primary adrenal insufficiency is a relatively rare disease. Isolated adrenal tuberculosis combined with adrenal insufficiency is a rare condition and may be difficult to distinguish from malignant tumors [1]. Adrenal failure due to tuberculosis mainly manifests itself as a clinical manifestation of primary adrenal insufficiency, the symptoms of which include generalized weakness, fatigue, anorexia, weight loss, and skin pigmentation. These symptoms manifest when more than $90 \%$ of the adrenal glands have been destroyed [2].

We report a case of suspected adrenal malignancy with adrenal insufficiency, which was finally diagnosed as adrenal tuberculosis.

\section{CASE REPORT}

A 40-year-old woman presented to the emergency department at Soonchunhyang University Gumi Hospital with altered consciousness. She had had weight loss and generalized weakness a year ago, and had irregular eating habits with decreasing appetite over two to three months. There were no specific findings on her past history and family history. At the time of admission, she was noted to present with a confused mental status, and her vital signs revealed a blood pressure of $115 / 71 \mathrm{~mm} \mathrm{Hg}$, pulse rate of 71/min, respiratory rate of $20 / \mathrm{min}$, and body temperature of $34.2^{\circ} \mathrm{C}$. Physical examination showed no abnormalities in her skin, limbs, and mouth. There was tenderness noted in her upper abdomen, but no hepatosplenomegaly. Lung auscultation and chest X-rays were negative for pathological signs.

Laboratory findings revealed fasting plasma glucose was significantly low at $22 \mathrm{mg} / \mathrm{dL}$. Other laboratory findings showed no abnormalities except for a slightly high serum amylase of $150 \mathrm{IU} / \mathrm{L}$ (Table 1). Urinalysis showed 3+glucose and ketone bodies. The rapid adrenocorticotropic hormone (ACTH) stimulation test revealed her cortisol was $0.65 \mu \mathrm{g} / \mathrm{dL}$ at baseline, $0.70 \mu \mathrm{g} / \mathrm{dL}$ after 30 minutes, and $0.77 \mu \mathrm{g} / \mathrm{dL}$ after 1 hour. Plasma ACTH was increased to $293.10 \mathrm{pg} / \mathrm{mL}$. 
Kim WS, et al. • Adrenal Tuberculosis with Primary Adrenal Insufficiency

Table 1. Laboratory findings

\begin{tabular}{lc}
\hline Variable & Result \\
\hline White blood cell count $\left(\times 10^{3} / \mu \mathrm{L}\right)$ & 8.310 \\
Hemoglobin $(\mathrm{g} / \mathrm{dL})$ & 14.0 \\
Platelet count $\left(\times 10^{3} / \mathrm{ML}\right)$ & 318 \\
Serum sodium $(\mathrm{mEq} / \mathrm{L})$ & 137 \\
Serum potassium $(\mathrm{mEq} / \mathrm{L})$ & 3.6 \\
Serum glutamic oxalate transaminase $(\mathrm{IU} / \mathrm{L})$ & 40 \\
Serum glutamic pyruvic transaminase $(\mathrm{IU} / \mathrm{L})$ & 27 \\
Plasma glucose $(\mathrm{mg} / \mathrm{dL})$ & 22 \\
Serum bilirubin, total $(\mathrm{mg} / \mathrm{dL})$ & 0.5 \\
Serum amylase $(\mathrm{IU} / \mathrm{L})$ & 150 \\
Blood urea nitrogen $(\mathrm{mg} / \mathrm{dL})$ & 16.9 \\
Serum creatinine $(\mathrm{mg} / \mathrm{dL})$ & 0.5 \\
\hline
\end{tabular}

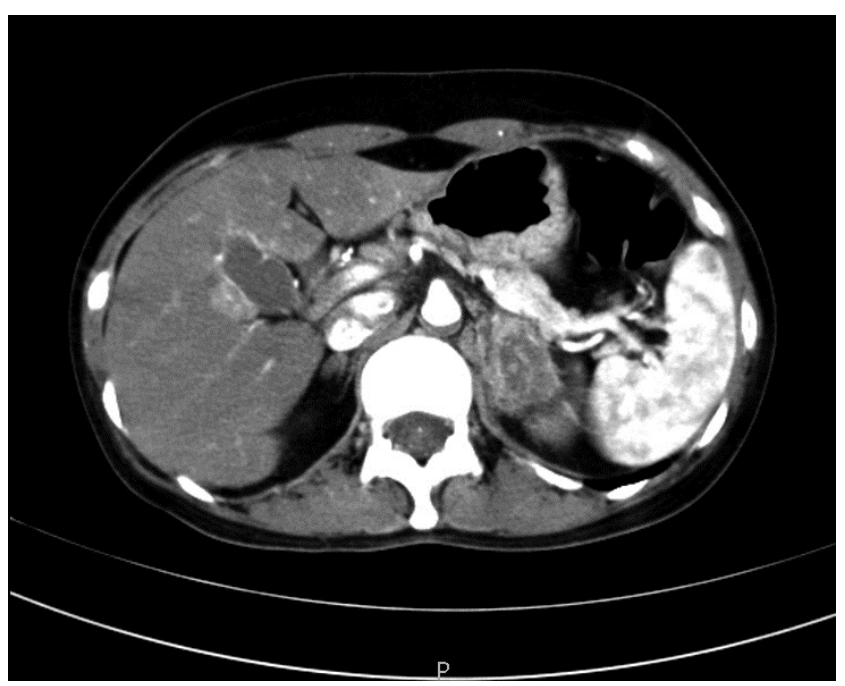

Fig. 1. Abdominal computed tomography revealed a calcified mass $3.3 \times 2.3 \mathrm{~cm}$ in size and peripheral enhancement of the left adrenal gland.

Abdominal computed tomography (CT) revealed a calcified mass $3.3 \times 2.3 \mathrm{~cm}$ in size and peripheral enhancement of the left adrenal gland (Fig. 1). There were no abnormal lesions noted in other abdominal and pelvic organs. There was no lymphadenopathy noted in the abdominal cavity, and a chest CT revealed no abnormalities.

Based on results of laboratory and radiological examination, she was diagnosed as having primary adrenal insufficiency and a left adrenal malignancy. She was initially treated with intravenous hydrocortisone and subsequently discharged on prednisone. After she showed significant improvement in her symptoms, we performed a laparoscopic left adrenalectomy, suspecting a malignancy of the adrenal glands. The patient was discharged on the 5th postoperative day without any complications. Microscopically, the

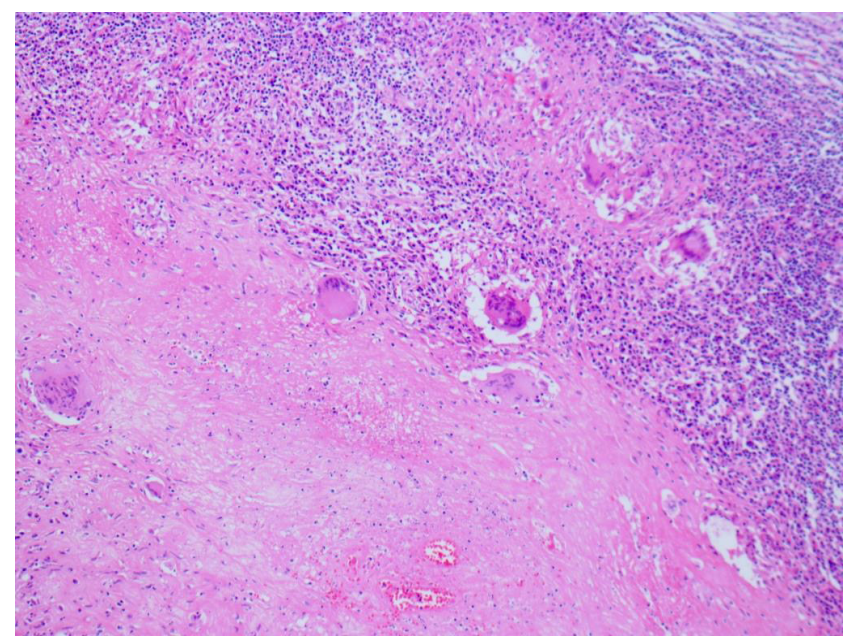

Fig. 2. Microscopically, the resected specimen revealed chronic granulomatous inflammation with central necrosis and multinucleated giant cells $(H \& E, \times 100)$.

resected specimen revealed chronic granulomatous inflammation with central necrosis and multinucleated giant cells (Fig. 2). The diagnosis of adrenal tuberculosis was confirmed using a final histological examination. The patient is being treatment with prednisone and anti-tuberculosis medication, and being followed up in the outpatient clinic.

\section{DISCUSSION}

Primary adrenal insufficiency is caused by destruction of adrenocortical tissue due to autoimmune disease, tuberculosis, cryptococcosis, fungal infection, and metastatic tumors, among other etiologies. The resulting adrenal gland destruction may manifest clinically as generalized fatigue, weight loss, and fever. In extreme situations, an 'adrenal crisis' may be precipitated as a result of infection, surgery or trauma, manifesting as low blood pressure, hyponatremia, and hypoglycemia [3]. Our patient had typical symptoms such as weight loss, generalized weakness, fatigue, and hypoglycemia. However, the reason she came to the emergency room due to altered consciousness was not due to adrenal crisis because correction of her blood sugar led to immediate improvement in her symptoms.

Tuberculosis may affect many of the endocrine glands including the hypothalamus, pituitary, and thyroid, but the most commonly involved endocrine organ is the adrenal gland. Mycobacterium tuberculosis complex spreads to the adrenal glands hematogenously. Adrenal involvement was found in $6 \%$ of patients with active tuberculosis in an autopsy series and adrenal tuberculosis was seen in 
$25 \%$ of these patients the adrenal gland was the only organ involved by tuberculosis. Adrenal biopsy is not necessary for primary adrenal insufficiency with bilateral adrenal enlargement in a patient with proven extra-adrenal tuberculosis. However, about $12 \%$ of patients with adrenal tuberculosis have no evidence of active extraadrenal tuberculosis. Adrenal biopsy is generally necessary in these patients to prove adrenal involvement by tuberculosis [4]. In our case, we did not perform adrenal biopsy because of unilateral adrenal enlargement and inability to reach the tumor via a safe path.

Typical radiological features of adrenal tuberculosis include bilateral enlargement of the adrenal glands showing peripheral enhancement and central necrotic areas, with or without calcifications [5]. As the disease advances, normal adrenal tissues are increasingly destroyed, resulting in adrenal insufficiency [6]. In our patient, adrenal tuberculosis could have been suspected on abdominal CT and by clinical examination. Adrenal biopsy is not necessary for primary adrenal insufficiency with bilateral enlargement of the adrenal glands in a patient with proven extra-adrenal tuberculosis. However, we selected laparoscopic adrenalectomy, which was performed for a definitive diagnosis because malignancy could not be completely excluded on studying the abdominal CT.

Initially described in 1992, laparoscopic adrenalectomy has rapidly become the gold standard of treatment for benign adrenal tumors [7]. The advantages over conventional open adrenalectomy include a lower complication rate, shorter hospital stay, reduced morbidity within 30 postoperative days, and lower overall costs. The laparoscopic approach has also been increasingly accepted for malignant adrenal tumors as well as large-sized benign lesions. As adrenal tumors increase in size, their malignant potential likewise increases, raising concerns for the use of laparoscopy for removal of large adrenal malignancies [8]. However, according to recent reports, no significant differences in the rate of recurrence or complications were seen when compared to an open procedure. Thus, laparoscopic adrenalectomy may also be performed for large and malignant tumors [9].

The adrenal gland is one of the organs with tuberculosis infects. In general, adrenal insufficiency due to adrenal tuberculosis almost involves both adrenal glands [10]. However, in our case, tuberculosis involved unilateral adrenal gland, resulting in adrenal insufficiency. This is a very rare case.

\section{REFERENCES}

1. Betterle C, Dal Pra C, Mantero F, Zanchetta R. Autoimmune adrenal insufficiency and autoimmune polyendocrine syndromes: autoantibodies, autoantigens, and their applicability in diagnosis and disease prediction. Endocr Rev 2002;23:327-64.

2. Nomura K, Demura H, Saruta T. Addison's disease in Japan: characteristics and changes revealed in a nationwide survey. Intern Med 1994;33: 602-6.

3. Kelestimur F. The endocrinology of adrenal tuberculosis: the effects of tuberculosis on the hypothalamo-pituitary-adrenal axis and adrenocortical function. J Endocrinol Investig 2004;27:380-6.

4. Kelestimur F, Unlu Y, Ozesmi M, Tolu I. A hormonal and radiological evaluation of adrenal gland in patients with acute or chronic pulmonary tuberculosis. Clin Endocrinol (Oxf) 1994;41:53-6.

5. Yang ZG, Guo YK, Li Y, Min PQ, Yu JQ, Ma ES. Differentiation between tuberculosis and primary tumors in the adrenal gland: evaluation with contrast-enhanced CT. Eur Radiol 2006;16:2031-6.

6. Ohwada S, Izumi M, Kawate S, Hamada K, Toya H, Togo N, et al. Surgical outcome of stage III and IV adrenocortical carcinoma. Jpn J Clin Oncol 2007;37:108-13.

7. Palazzo FF, Sebag F, Sierra M, Ippolito G, Souteyrand P, Henry JF. Longterm outcome following laparoscopic adrenalectomy for large solid adrenal cortex tumors. World J Surg 2006;30:893-8.

8. Donatini G, Caiazzo R, Do Cao C, Aubert S, Zerrweck C, El-Kathib Z, et al. Long-term survival after adrenalectomy for stage I/II adrenocortical carcinoma (ACC): a retrospective comparative cohort study of laparoscopic versus open approach. Ann Surg Oncol 2014;21:284-91.

9. Machado NO, Al Qadhi H, Al Wahaibi K, Rizvi SG. Laparoscopic adrenalectomy for large adrenocortical carcinoma. JSLS 2015;19:e2015.00036.

10. Ikoma A, Namai K, Saito T, Kawano T, Saito T, Kasono K, et al. Unilateral active adrenal tuberculosis featuring persistent intermittent fever. Endocr J 2004;51:463-6. 